\title{
CORRIGENDUM
}

\section{Loci influencing blood pressure identified using a cardiovascular gene-centric array}

Santhi K. Ganesh, Vinicius Tragante, Wei Guo, Yiran Guo, Matthew B. Lanktree, Erin N. Smith, Toby Johnson, Berta Almoguera Castillo, John Barnard, Jens Baumert, Yen-Pei Christy Chang, Clara C. Elbers, Martin Farrall, Mary E. Fischer, Nora Franceschini, Tom R. Gaunt, Johannes M.I.H. Gho, Christian Gieger, Yan Gong, Aaron Isaacs, Marcus E. Kleber, Irene Mateo Leach, Caitrin W. McDonough, Matthijs F.L. Meijs, Olle Mellander, Cliona M. Molony, Ilja M. Nolte, Sandosh Padmanabhan, Tom S. Price, Ramakrishnan Rajagopalan, Jonathan Shaffer, Sonia Shah, Haiqing Shen, Nicole Soranzo, Peter J. van der Most, Erik P.A. Van Iperen, Jessica Van Setten, Judith M. Vonk, Li Zhang, Amber L. Beitelshees, Gerald S. Berenson, Deepak L. Bhatt, Jolanda M.A. Boer, Eric Boerwinkle, Ben Burkley, Amber Burt, Aravinda Chakravarti, Wei Chen, Rhonda M. CooperDeHoff, Sean P. Curtis, Albert Dreisbach, David Duggan, Georg B. Ehret, Richard R. Fabsitz, Myriam Fornage, Ervin Fox, Clement E. Furlong, Ron T. Gansevoort, Marten H. Hofker, G. Kees Hovingh, Susan A. Kirkland, Kandice Kottke-Marchant, Abdullah Kutlar, Andrea Z. LaCroix, Taimour Y. Langaee, Yun R. Li, Honghuang Lin, Kiang Liu, Steffi Maiwald, Rainer Malik, CARDIOGRAM METASTROKE, Gurunathan Murugesan, Christopher Newton-Cheh, Jeffery R. O’Connell, N. Charlotte Onland-Moret, Willem H. Ouwehand, Walter Palmas, Brenda W. Penninx, Carl J. Pepine, Mary Pettinger, Joseph F. Polak, Vasan S. Ramachandran, Jane Ranchalis, Susan Redline, Paul M. Ridker, Lynda M. Rose, Hubert Scharnag, Nicholas J. Schork, Daichi Shimbo, Alan R. Shuldiner, Sathanur R. Srinivasan, Ronald P. Stolk, Herman A. Taylor, Barbara Thorand, Mieke D. Trip, Cornelia M. van Duijn, W. Monique Verschuren, Cisca Wijmenga, Bernhard R. Winkelmann, Sharon Wyatt, J. Hunter Young, Bernhard O. Boehm, Mark J. Caulfield, Daniel I. Chasman, Karina W. Davidson, Pieter A. Doevendans, Garret A. FitzGerald, John G. Gums, Hakon Hakonarson, Hans L. Hillege, Thomas Illig, Gail P. Jarvik, Julie A. Johnson, John J.P. Kastelein, Wolfgang Koenig, LifeLines Cohort Study, Winfried März, Braxton D. Mitchell, Sarah S. Murray, Albertine J. Oldehinkel, Daniel J. Rader, Muredach P. Reilly, Alex P. Reiner, Eric E. Schadt, Roy L. Silverstein, Harold Snieder, Alice V. Stanton, André G. Uitterlinden, Pim van der Harst, Yvonne T. van der Schouw, Nilesh J. Samani, Andrew D. Johnson, Patricia B. Munroe, Paul I.W. de Bakker, Xiaofeng Zhu, Daniel Levy, Brendan J. Keating and Folkert W. Asselbergs

Human Molecular Genetics 2013; 22:8; pp. 1663-1678; doi:10.1093/hmg/dds555

An author's name was incorrectly shown as Jessic A. Van Setten when it should have been Jessica Van Setten as shown above. Also, in the Replication Cohorts section of Table 1, we have noticed some errors in the medication column. The corrected Table is shown here.

The authors apologise for these mistakes.

(C) The Author 2013. Published by Oxford University Press. All rights reserved.

For Permissions, please email: journals.permissions@oup.com 
Table 1: Clinical Characteristics

\begin{tabular}{|c|c|c|c|c|c|c|c|c|}
\hline Discovery Cohorts & Age & Female/Male & SBP & DBP & MAP & PP & BMI & $\begin{array}{l}\text { Taking anti-hypertensive } \\
\text { medication }\end{array}$ \\
\hline AMC-PAS & $42.9 \pm 5.3$ & $180 / 563$ & $128.6 \pm 17.9$ & $79.9 \pm 10.7$ & $96.1 \pm 12.1$ & $48.8 \pm 13.1$ & $26.9 \pm 4.1$ & $33.2 \%$ \\
\hline Amish & $47.6 \pm 15.0$ & $713 / 691$ & $121.9 \pm 16.5$ & $75.3 \pm 9.4$ & $90.8 \pm 10.8$ & $46.6 \pm 12.2$ & $27.3 \pm 5.0$ & $16.4 \%$ \\
\hline ARIC & $54.2 \pm 5.7$ & $5124 / 4453$ & $118.3 \pm 17$ & $71.5 \pm 10$ & $87.1 \pm 11.3$ & $46.7 \pm 12.7$ & $26.9 \pm 4.9$ & $25.1 \%$ \\
\hline BHS & $22.5 \pm 4.4$ & $291 / 228$ & $111.5 \pm 10.2$ & $71.8 \pm 8.5$ & $85 \pm 8.3$ & $39.7 \pm 8$ & $24.7 \pm 6.1$ & $2.7 \%$ \\
\hline CARDIA & $40.6 \pm 4.1$ & $703 / 623$ & $102.2 \pm 30.5$ & $71.9 \pm 11.2$ & $84.5 \pm 11.4$ & $37.7 \pm 8.8$ & $26.9 \pm 6.4$ & $3.7 \%$ \\
\hline CCCS & $64.2 \pm 9.7$ & $555 / 1402$ & $136.94 \pm 19.04$ & $78.33 \pm 10.61$ & $156.48 \pm 23.57$ & $58.61 \pm 15.69$ & $29.2 \overline{(4.8) / 29.8(6.5)}$ & $88.7 \%$ \\
\hline CFS & $40.9 \pm 19.9$ & $302 / 252$ & $121.1 \pm 16.6$ & $71.8 \pm 11.7$ & $88.2 \pm 12.2$ & $49.3 \pm 12.4$ & $30.1 \pm 8.8$ & $8.8 \%$ \\
\hline CHS & $72.6 \pm 6.3$ & $2208 / 1722$ & $135.3 \pm 21.5$ & $69.9 \pm 11.6$ & $91.7 \pm 12.9$ & $65.4 \pm 18.6$ & $26.3 \pm 4.8$ & $39.7 \%$ \\
\hline CLEAR & $67.8 \pm 9.6$ & $0 / 1365$ & $151.4 \pm 22.4$ & $82.2 \pm 12.3$ & $105.3 \pm 13.8$ & $69.2 \pm 18.4$ & $28.1 \pm 5.0$ & $67.0 \%$ \\
\hline EPIC_NL & $54.06 \pm 10.11$ & $4057 / 1137$ & $133.13 \pm 21.22$ & $80.46 \pm 10.93$ & $97.98 \pm 13.20$ & $52.65 \pm 15.68$ & $26.77 \pm 4.45$ & $0 \%$ \\
\hline FHS & $40.9 \pm 9.1$ & $3775 / 3134$ & $118.6 \pm 14.3$ & $76.4 \pm 9.8$ & $90.5 \pm 10.6$ & $42.2 \pm 9.4$ & $26.1 \pm 5$ & $5.5 \%$ \\
\hline GIRaFH & $44.5 \pm 11.7$ & $882 / 812$ & $134.9 \pm 19.2$ & $82.0 \pm 10.5$ & $99.7 \pm 12.3$ & $52.9 \pm 14.4$ & $25.1 \pm 3.5$ & $9.3 \%$ \\
\hline GQ2 & $65.5 \pm 10.5$ & $385 / 93$ & $130.51 \pm 22.40$ & $72.71 \pm 12.89$ & $149.78 \pm 27.66$ & $57.81 \pm 18.47$ & $29.7 \overline{(7.7) / 29.5(6.3)}$ & $76.2 \%$ \\
\hline INVEST & $69.4 \pm 9.5$ & $467 / 580$ & $160.8 \pm 17.4$ & $90.6 \pm 10.1$ & $114.0 \pm 10.6$ & $70.1 \pm 15.9$ & $29.0(4.7) / 28.4(6.2)$ & $82.6 \%$ \\
\hline LURIC & $58.1 \pm 8.6$ & $558 / 1480$ & $151.2 \pm 24.4$ & $89.8 \pm 12.1$ & $110.3 \pm 15.0$ & $61.4 \pm 17.6$ & $27.7 \pm 4.2$ & $85.6 \%$ \\
\hline MEDAL & $62.9 \pm 9.0$ & $1178 / 2820$ & $136.8 \pm 16.0$ & $81.7 \pm 9.6$ & $94.9 \pm 8.6$ & $52.9 \pm 11.5$ & $30.5 \pm 6.3$ & $1.4 \%$ \\
\hline MESA & $62.7 \pm 10.3$ & $1199 / 1097$ & $123.5 \pm 20.8$ & $70.1 \pm 10.2$ & $87.9 \pm 12.3$ & $53.4 \pm 16.7$ & $27.8 \pm 5.1$ & $33.3 \%$ \\
\hline MONICA/KORA F3 & $57.6 \pm 8.1$ & $755 / 649$ & $131.8 \pm 19.4$ & $83.3 \pm 10.3$ & $99.5 \pm 12.6$ & $48.5 \pm 13.2$ & $27.8 \pm 4.5$ & $30.1 \%$ \\
\hline MONICA/KORA S12 & $51.8 \pm 9.9$ & $431 / 549$ & $133.6 \pm 19.1$ & $81.5 \pm 11.1$ & $98.8 \pm 12.5$ & $52.1 \pm 14.6$ & $27.2 \pm 4.0$ & $16.6 \%$ \\
\hline NSHS95 & $49.4 \pm 18.4$ & $857 / 899$ & $126.6 \pm 17.7$ & $76.7 \pm 11.6$ & $93.3 \pm 11.8$ & $49.9 \pm 15.8$ & $27.1 \pm 5.5$ & N/A \\
\hline PEAR & $50.1 \pm 9.4$ & $194 / 244$ & $151.8 \pm 12.4$ & $98.0 \pm 5.7$ & $115.9 \pm 6.9$ & $53.7 \pm 10.8$ & {$[30.3(4.4) / 30.4(6.1)]$} & $0 \%$ \\
\hline PennCAC & $56.0 \pm 8.0$ & $631 / 1145$ & $132 \pm 23.2$ & $72.4 \pm 11.2$ & $52.6 \pm 12.6$ & $59.5 \pm 19.7$ & $29.8 \pm 5.9$ & N/A \\
\hline PennCath & $52.0 \pm 9.0$ & $739 / 1386$ & $127 \pm 15.1$ & $76.7 \pm 9.5$ & $62.9 \pm 10.5$ & $51.9 \pm 12.3$ & $30.1 \pm 5.9$ & $32.8 \%$ \\
\hline SMART & $59.36 \pm 12.25$ & 206/299 & $158.64 \pm 18.57$ & $94.76 \pm 11.80$ & $116.06 \pm 12.87$ & $63.88 \pm 13.78$ & $27.35 \pm 4.62$ & $39.4 \%$ \\
\hline WHI & $68.0 \pm 6.6$ & $7606 / 0$ & $133.0 \pm 18.8$ & $75.0 \pm 9.7$ & $94.3 \pm 11.0$ & $58.0 \pm 16.2$ & $28.3(6.2)$ & $33.3 \%$ \\
\hline Replication Cohorts & Age & Female/Male & SBP & DBP & MAP & PP & BMI & $\begin{array}{l}\text { Taking anti-hypertensive } \\
\text { medication }\end{array}$ \\
\hline AIBIII & $52.8(9.2)$ & 249/209 & $119.9(13.7)$ & $75.4(7.6)$ & $90.2(8.8)$ & $44.5(10.3)$ & $25.7(3.6)$ & $0.0 \%$ \\
\hline ASCOT & $63(8.1)$ & $224 / 1015$ & $161.4(17.8)$ & $92.9(9.9)$ & $115.7(10.6)$ & $68.5(16.1)$ & $29.1(4.6)$ & $89.2 \%$ \\
\hline BRIGHT (controls) & $58.7(8.9)$ & $1088 / 647$ & $123(10.5)$ & $76.4(7.2)$ & $91.9(7.5)$ & $46.7(8.3)$ & $25.3(3.3)$ & $0.0 \%$ \\
\hline BRIGHT (cases) & $58(10.3)$ & $1144 / 775$ & $154.3(21.1)$ & $93.9(11.3)$ & $114(13.3)$ & $60.4(15.7)$ & $58(10.3)$ & $92.5 \%$ \\
\hline BWHHS & $68.85(5.51)$ & $3373 / 0$ & $146.53(26.59)$ & $79.16(12.85)$ & $102(15)$ & $67.6(19)$ & $27.25(5.95)$ & $30.2 \%$ \\
\hline GRAPHIC & $39.30(14.50)$ & $1004 / 1020$ & $127.09(17.84)$ & $79.12(10.96)$ & $95.1(12.5)$ & $48(11.9)$ & $26.11(4.61)$ & $6.7 \%$ \\
\hline LIFELINES & $47.3 \pm 11.2$ & $4640 / 3483$ & $127.9 \pm 15.7$ & $75.1 \pm 9.1$ & $52.7 \pm 11.8$ & $92.7 \pm 10.3$ & $26.3 \pm 4.3$ & $15.5 \%$ \\
\hline MDC & $57.8(5.9)$ & $1074 / 772$ & $115.6 \overline{(5.8)}$ & $73.6(5.3)$ & $87.6(4.7)$ & $42(6)$ & $24.3(3.3)$ & $0.0 \%$ \\
\hline NBS & $41.36(12.37)$ & $1183 / 1169$ & N/A & N/A & N/A & N/A & N/A & N/A \\
\hline NESDA & $41.5 \pm 12.7$ & $1166 / 551$ & $135.3 \pm 20.2$ & $81.7 \pm 11.8$ & $99.6 \pm 13.9$ & $53.6 \pm 12.7$ & $25.5 \pm 4.9$ & $12.8 \%$ \\
\hline NORDIL & $56(4)$ & 979/940 & $177.3(14.6)$ & $105.9 \overline{(5.5)}$ & $129.7 \overline{(7.1)}$ & $71.5 \overline{(13.9)}$ & $28.3(4.6)$ & $0.0 \%$ \\
\hline PREVEND & $49.6 \pm 12.5$ & $1752 / 1869$ & $129.1 \pm 19.9$ & $74.1 \pm 9.9$ & $54.9 \pm 13.9$ & $92.4 \pm 12.5$ & $26.1 \pm 4.3$ & $14.2 \%$ \\
\hline Procardis & $59.34(9.93)$ & $1634 / 1564$ & $130.75(17.11)$ & $79.63(10.03)$ & $96.7(11.2)$ & $51.1(13.4)$ & $26.81(4.37)$ & $19.3 \%$ \\
\hline Rotterdam Study & $69.4(9.1)$ & $3327 / 2327$ & $144.1(24.2)$ & $76.9(12.6)$ & $99.3(15.0)$ & $67.1(18.5)$ & $26.3(3.7)$ & $32.5 \%$ \\
\hline TRAILS clinical cohort & $15.8 \pm 0.6$ & $97 / 217$ & $119.1 \pm 12.6$ & $61.0 \pm 6.6$ & $80.4 \pm 7.3$ & $58.1 \pm 11.4$ & $21.5 \pm 3.6$ & N/A \\
\hline TRAILS population cohort & $16.2 \pm 0.7$ & $693 / 642$ & $118.1 \pm 12.4$ & $61.1 \pm 6.9$ & $80.1 \pm 7.4$ & $57.7 \pm 10.6$ & $21.2 \pm 3.2$ & N/A \\
\hline WGHS & $54.2 \pm 7.1$ & $22625 / 0$ & $125.5 \pm 16.4$ & $78.0 \pm 10.7$ & $93.8 \pm 11.9$ & $47.6 \pm 10.4$ & $25.9 \pm 5.0$ & $12.9 \%$ \\
\hline WHII & $60.83 \overline{(6.0)}$ & $1845 / 3210$ & $128.1(16.7)$ & $74.6 \overline{(10.5)}$ & $92.4 \overline{(11} .8)$ & $53.5 \overline{(11.2)}$ & $26.7 \overline{(4.3)}$ & $22.8 \%$ \\
\hline
\end{tabular}

Mean \pm Standard deviation is given for each phenotype, except $\%$ where indicated. 